\title{
LV. On focal lines, and anchor-ring wave-fronts
}

\section{Prof. J.D. Everett F.R.S.}

To cite this article: Prof. J.D. Everett F.R.S. (1902) LV. On focal lines, and anchor-ring wavefronts, Philosophical Magazine Series 6, 3:17, 483-486, DOI: $10.1080 / 14786440209462792$

To link to this article: http://dx.doi.org/10.1080/14786440209462792

曲 Published online: 15 Apr 2009.

Submit your article to this journal 준

Џll Article views: 2

Q View related articles $₫$ 
LV. On Focal Lines, and Anchor-Ring Wave-Fronts.

By Prof. J. D. EvereTr, F.R.S.*

WHEN a small cone of rays is obliquely incident on a spherical reflecting or refracting surface, the rays after reflection or refraction no longer compose a true cone. Instead of meeting in a point they form a narrow neck; and this neck is flattened in two places called the primary and secondary foci, the planes of flattening heing at right angles to each other. Optical writers give the name focal lines to the sectious of the pencil made at these two places by planes perpendicular to the axis of the pencil ; but it would be more appropriate to give the name to the sections which must nearly resemble lines, whatever angle they may make with the axis of the pencil.

Clearness of conception, in intricate matters, is greatly aided by sharply defined illustration; and I wish to call attention to a case (which appears to have been hitberto overlooked) in which all the rays, even of a large pencil, pass accurately through two definite lines : one of these lines being a circular arc cutting the pencil at right angles; and the other being a straight line, which may have any inclination to the axis of the pencil.

The case is that in which the wave-front in one of its positions is a tore (or anchor-ring).

A tore may be defined as the surface generated by the revolution of a circle round a fixed straight line in its plane; this line we shall refer to as the axis of revolution. A tore

Fig. 1.

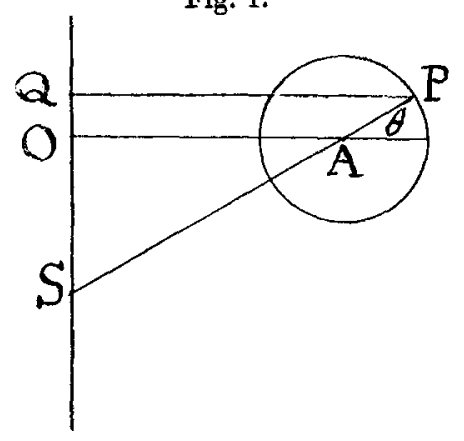

has also what may be called a circular axis - the circle generated by the motion of the centre of the revolving circle. In the figure QOS is the axis of revolution, $D$ the centre of the tore, and $\mathrm{OA}$ the radius of the circular axis. $P$ is any

* Communicated by the Physical Suciety : read February 28, 1902. 
point on the revolving circle, $\mathrm{PQ}$ a perpendicular on the axis of revolution, and $\theta$ the inclination of the straight line PAS to $\mathrm{PQ}$ or $\mathrm{AO}$.

The two focal lines are always at the centres of curvature of two mutually rectangular normal sections of the wavefront, one being the section of greatest and the other of least radius of curvature. For an element of the wave-front at $P$ one of these sections is the circle shown in our diagram, and $\mathrm{PA}$ is its radius of curvature. The other is the section of the tore made by a plane through PAS perpendicular to the plane of the diagram. To find its radius of curvature note that $\mathrm{QP}$ is the radius of a circular section made by a plane perpendicular to the axis QS ; hence, by Meunier's theorem, the required radius of curvature is $P Q \sec \theta$, that is $P S$. The two focal lines are accordingly at $A$ and $S$. $A$ is called the primary, and $\mathrm{S}$ the secondary focus. If we make $\mathrm{P}$ travel round the circle shown in the figure the primary focus remains fixed at $A$, and the secondary focus travels along the axis $Q S$, its distance from $\mathrm{O}$ being $\mathrm{OA} \sec \theta$, which runs from zero to infinity in both directions. On the other hand, if we make $P$ revolve round the axis $Q S$ the secondary focus remains fixed at $S$, and the primary focus generates the circular axis of the tore.

For a circular element of the wave-front, of small diameter $d$, having $\mathrm{P}$ for its centre, the primary focal line will be an arc of the circular axis, of length $\frac{\mathrm{SA}}{\mathrm{SP}} d$; and the secondary focal line will be a portion of the axis of revolution, of length $\frac{\mathrm{AS}}{\mathrm{AP}} d \cdot \sec \theta$. If, instead of regarding this absolutely sharp line as the focal line, we follow the usual convention, and adopt, as the secondary line, the section of the pencil by a plane at $S$ perpendicular to $P S$, its two ends will be blurred, so that it will resemble a figure of 8 , and its length will be AS $\frac{\mathrm{AS}}{\mathrm{AP}} d$.

At any point ' $\mathrm{I}$ ' between $\mathrm{A}$ and $\mathrm{S}$ the breadth of the pencil in the plane of the diagram is $\frac{\mathrm{AT}}{\mathrm{AP}} d$, and its breadth perpendicular to the plane of the diagram is $\frac{\mathbf{S T}}{\mathbf{S P}} d$. The ratio of the latter breadth to the former is $\frac{\mathrm{ST}}{\mathrm{AT}}$ multiplied by the constant $\frac{\mathrm{AP}}{\mathrm{SP}}$, and is unity wher $\mathrm{SA}$ is divided internally and externally in the same ratio. 
Toric wave-fronts can be produced by constructing a concave reflector, of the form generated by making an ellipse, or any portion of an ellipse, revolve through any convenient angle round an ordinate erected at one focus $\mathrm{O}$ of the ellipse; and employing it to reflect rays diverging from a small source of light at this focus. The length of path is the same for all once-reflected rays from $\mathrm{O}$ to the circle traced by the other focus A, and will, therefore, be the same for all such rays from $\mathrm{O}$ to a tore having this circular axis.

Figs. 2, 3, 4 represent an experimental illustration. Fig. 2

Fig. 2.

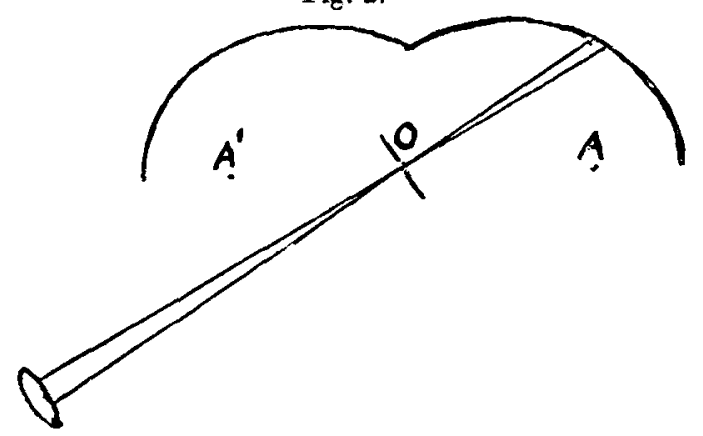

contains an axial section of a reflecting surface of revolution; the section being made up of portions of two equal ellipses of eccentricity $\frac{1}{2}$. $\mathrm{O}$ and $\mathrm{A}, \mathrm{A}^{\prime}$ are points corresponding to $\mathrm{O}$ and $A$ in fig. 1 . A point source at $O$ is provided by mean. of an opaque disk pierced with a small hole at $O$; the disk being carried by an axis in its own plane, coinciding with a diameter of the bounding circle of the reflector, as shown in figs. $3,4(\mathrm{p} .486)$, which represent the reflector supported on a stand, with its bounding plane vertical, the axis of rotation of the disk being also vertical. By rotation round this axis the disk can be made to face a source of light at its own level, at any obliquity to the bounding plane. In fig. 2 a beam is shown focussed on the hole by a lens. The beam may be furnished by a lantern, or by a bright lamp-flame properly screened. Widening the angle of the pencil given by the lens lengthens and at the same time time brightens the focal lines.

The primary line will be a circle having $\mathbf{A ~ A}^{\prime}$ for diameter, and will be always real. The secondary line will coincide with the axis of revolution, and will be real or virtual according to the position of the area of incidence. For incidence at the extremily of the ordinate throngh $\mathbf{A}$, the reflected ray is parallel to the axis, and the secondury line goes off to 
infinity. Both lines when real can be shown on a translucent screen. The secondary line, when virtual, can be seen by looking into the reflector. The complete circle of the primary

Fig. 3.

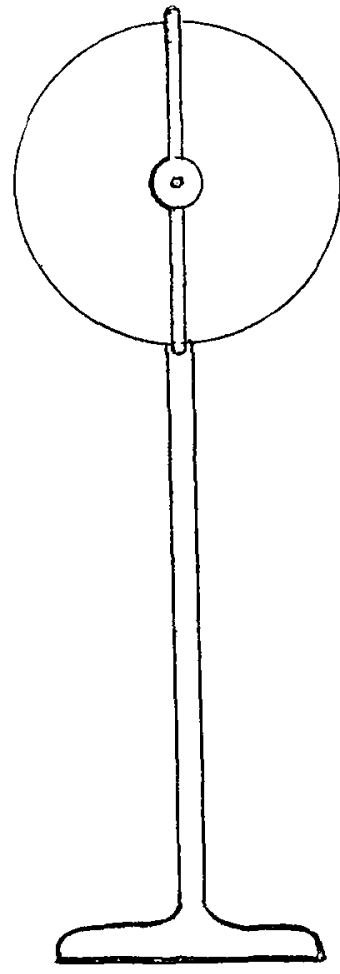

Fig. 4.

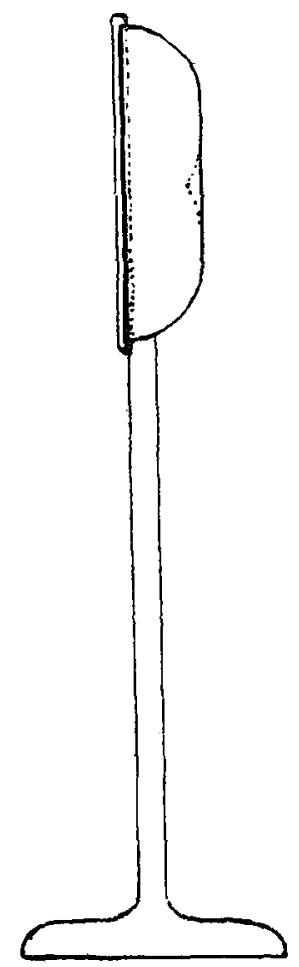

can be obtained at once by making the axis of the incident beam (of wide angle) coincide with the axis of the reflector, so that the area of incidence surrounds the central boss.

An iris diaphragm (as used by microscopists) might with advantage be substituted for the pierced disk.

Postseript. - Since reading the paper I have found that Maxwell, in a paper entitled "On the Cyelide," has investigated the general form of the wave-surface "when one or both of the so-called tocal lines is really a line," and has indicated the anchor-ring as a particular case. ("Collected Papers,' vol. ii. pp. 144 \& 151.) 